\title{
Etiology and Epidemiology of Catheter Related Bloodstream Infections in Patients Receiving Home Parenteral Nutrition in a Gastromedical Center at a Tertiary Hospital in Denmark
} \author{
Gyldenlykke ${ }^{4}$, Michael Tvede ${ }^{2}$ and Leif Percival Andersen ${ }^{3}$. \\ ${ }^{1}$ Department of Clinical Microbiology, Slagelse Hospital, Slagelse, Denmark \\ ${ }^{2}$ Department of Clinical Microbiology, University Hospital of Copenhagen, Denmark \\ ${ }^{3}$ Department of Infection Control, University Hospital of Copenhagen, Denmark \\ ${ }^{4}$ Department of Gastroenterology, University Hospital of Copenhagen, Copenhagen, Denmark
}

Xiaohui Chen Nielsen ${ }^{1,2, *}$, Ming Chen ${ }^{2}$, Anne-Marie Blok Hellesøe ${ }^{3}$, Palle Bekker Jeppesen ${ }^{4}$, Jonna

\begin{abstract}
We conducted a retrospective epidemiologic study of catheter related bloodstream infections (CRBSI) in patients receiving long-term home parenteral nutrition (HPN) from January 2002 to December 2005. Our results showed that coagulase negative staphylococci (CoNS) were the most prevalent pathogens (44.7\% of all CRBSI episodes), followed by Enterobacteriaceae (33.2\%). Prevalence for candidemia and Enterococcus bacteremia was relatively high (14.4\% and $10.8 \%$, respectively). Cefuroxime resistance was observed in $65.4 \%$ CoNS and $31.5 \%$ Enterobacteriaceae. Based on the results from the study, a new empiric antimicrobial treatment regiment was suggested.
\end{abstract}

Keywords: Etiology, epidemiology, intravascular catheter, blood stream infection, home parenteral nutrition, antimicrobial susceptibility.

\section{INTRODUCTION}

Parenteral nutrition $(\mathrm{PN})$ represents a lifesaving treatment for patients with severe intestinal failure. Its use at home (HPN) was introduced in 1967 [1] and is now a commonly used procedure [2]. The most frequent HPN complication is catheter related bloodstream infection (CRBSI), which is an important factor for morbidity and hospital costs for the patients receiving HPN [3, 4]. Initial antibiotic treatment is crucial for infection outcome. Therefore, empiric treatment for CRBSI in patients receiving HPN should be guided primarily by local epidemiological data. We have surveyed the incidence of CRBSI, risk factors and predominant pathogenic organisms for CRBSI in patients receiving HPN. The efficacy of initial empirical treatment with cefuroxime, gentamicin and metronidazole was also evaluated.

\section{MATERIAL AND METHODS}

We conducted a retrospective, descriptive, observational epidemiologic study of patients receiving long-term HPN from January 2002 to December 2005 in the Department of Medical Gastroenterology at a tertiary university hospital in Denmark.

\footnotetext{
*Address correspondence to this author at the Department of Clinical Microbiology, Slagelse Hospital, Region Zealand, Ingemannsvej 18, DK-4200 Slagelse, Denmark; Tel: +45 5855 9404, Fax: +45 5855 9410,

E-mail: xcn@regionsjaelland.dk
}

Patients: The positive blood culture data from the Department of Medical Gastroenterology between 2002 and 2005 were extracted from the microbiological databaseMADS. The patients who did not receive HPN were excluded.

Information about age, gender, number of CRBSI, microbiologic characteristics, underlying disease, co-morbidity, caring methods, catheter type, complications to HPN, concurrent infections, duration of antibiotic treatment, and clinical outcome were registered for each patient after reviewing the charts. Infection parameters, total leukocyte count (LCC), C-reactive protein (CRP), were collected from the clinical biochemical department database.

Bacteremia episodes: When isolation of the same bacteria in blood culture occurred more than once in the patient within a period of 30 days, it was regarded as one episode.

The bacterial cultures were identified by automatic system VITEK II (BioMérieux) according to the manufactures instructions. When yeast is identified from the culture medium, the species identification is performed by incubating the yeast culture on chromID Candida agar (BioMérieux).

Statistical analysis: Multi-variant analysis was applied for the risk factors analysis. The examined risk factors were underlying disease, co-morbidity, HPN caring person, complications related to HPN, and concurrent infections. The statistic program used was SPSS. 
The project is approved by DataTilsynet in Denmark.

\section{RESULTS}

A total of 216 patients receiving HPN were registered in the Department of Medical Gastroenterology between 2002 and 2005. The inclusion criteria for the patient to go with parenteral support is intestinal failure, i.e. the inability to maintain sufficient fluid, electrolyte, micro- or macronutrient balance when on oral or enteral nutrtition.

All patients had an uncoated one-lumen silicone rubber catheter Broviac catheter (Bard Limited, Crawley, UK).

All patients follow the standard operational procedure of the department.

There were no restrictions for the physical activities. However, most of the patients had limited physical activities and more sedentary life because of their disease. Only $20 \%$ of patients could continue their professional occupation.

The most frequent primary disease for patients receiving HPN was Crohns disease (27.8\%), followed by arte$\mathrm{rial} /$ venous mesenteric infarction $(24.7 \%)$ and radiation enteritis $(19.6 \%)$.

$54.6 \%$ of the patients handled the HPN by themselves, $5.2 \%$ patients had family members to handle the HPN, while $27.8 \%$ patients had a care assistant to handle the HPN (Table 1).

Table 1. Demographical and Clinical Characteristics for the 97 Patients with CRBSI in the Period 2002-2005

\begin{tabular}{|l|c|}
\hline \multicolumn{1}{|c|}{ Characteristics } & Cohort \\
\hline \hline Sex, number of patients & \\
Male: female & $38: 59$ \\
\hline Age, years & \\
Mean (range) & $54.2(18-87)$ \\
\hline Primary disease, number (\%) & \\
Chrohn's disease & $27(27.8)$ \\
Colitis ulcerosa & $8(8,3)$ \\
Arteial/venous mesenteric infarction & $24(24.7)$ \\
Radiation enteritis & $19(19,6)$ \\
Miscellaneous & $19(19.6)$ \\
\hline Co-morbidity, number (\%) & \\
Diabetes mellitus & $10(4.4)$ \\
Immunosuppression ${ }^{\mathrm{a}}$ & $10(4.4)$ \\
Others & $15(16.5)$ \\
\hline HPN caring, number (\%) & \\
Patient self & $53(54.6)$ \\
Patient's family & $27(27.8)$ \\
Caregiver & $5(5.2)$ \\
Patient self and caregiver/family member & $12(12.4)$ \\
\hline
\end{tabular}

a.Patients who had received chemotherapy or radiation therapy in the past one month, and/or if the total leukocyte count is less than $1.5 \mathrm{million} / \mathrm{ml}$ were considered as immunosuppressed.
225 ICRB episodes from 97 patients were identified by the microbiological database-MADS and the patients clinical chart. The incidence of CRBSI was $44.9 \%$.

Fifty out of 97 patients (51\%) had more than one CRBSI episode. Polymicrobial infections were registered in thirtyseven out of 225 episodes (16.4\%). CRBSI occurred after 30 days of catheter installation in $92.9 \%$ of episodes, while CRBSI occurred after one year of catheter installation comprised $61.4 \%$ of the patients. Fifteen patients died during the observation period, while no death related to CRBSI among the patients on HPN was observed.

The catheters were preserved as long as it is functional. When CRBSI occurs, preservation of the catheter will be attempted with relevant antimicrobial therapy. Recurrent infections and sepsis related to CRBSI are two of the indications for the removal of a catheter.

The distribution of the predominant microorganism based on infection episodes were:

Coagulase negative Staphylococci (CoNS) appeared in $44.7 \%$ CRBSI episodes (43 patients). Enterobacteriaceae appeared in $33.2 \%$ (47 patients), Candida spp. in $14.4 \%$ (18 patients), Enterococcus spp. in 10.8\% (13 patients), and $S$. aureus in $9.2 \%$ (15 patients). Anaerobic bacteria were found only in 1.3\% (3 patients). Among Candida spp., 38.6\% (13 patients) had C. albicans, $31.8 \%$ (9 patients) had $C$. glabrata, while in 5 patients both Candida spp. were found. It was not possible to evaluate the infections were nosocomial or community acquired, since the patients were in the hospital surroundings very often. The distribution of predominant microorganisms for each year is shown in Table 2.

\section{Antimicrobial susceptibility:}

Among CoNS, $65.4 \%$ were methicillin resistant, and $31.6 \%$ were resistant to both methicillin and gentamicin. All S. aureus isolates were sensitive to methicillin and gentamycin. No MRSA was observed in the period. Among Enterococcus spp., $37.8 \%$ isolates were resistant to carbapenems. All Staphylococcus and Enterococcus isolates were susceptible to vancomycin. Among Enterobacteriaceae, 9.2\% were ciprofloxacin resistant, $10.6 \%$ were gentamicin resistant, $31.5 \%$ were cefuroxime resistant, and $11.4 \%$ were resistant to ceftriaxon. Only $4 \%$ were resistant to both cefuroxime and gentamicin. The presence of extended $\beta$-lactamase was not investigated since it was not part of the routine procedure. All Enterobacteriaciae isolates were susceptible to carbarpenem.

Combination of intravenous cefuroxime, gentamicin and metronidazole was the standard empirical antimicrobial therapy regiment in this department and this combination was used as initial treatment for $61.1 \%$ of the CRBSI episodes. Cefuroxime, ciprofloxacin and metronidazole was the initial treatment in $12.4 \%$ episodes. All patients received antibiotic treatment at duration of 6-14 days. None of the examined risk factors were associated with higher risk for relapse or recurrent infections or any specific microorganism based on multi-variant analysis (SPSS).

\section{DISCUSSIONS}

The incidence of CRBSI varies from studies to studies [5-7]. In this study, $44.9 \%$ of all patients receiving HPN in 
Table 2. Epidemiology of Microorganisms Causing Catheter-related Bloodstream Infections in Patients Receiving Home Parenteral Nutrition (HPN) from Year 2002 to 2005 (Some of the Episodes were Multi-microbial)

\begin{tabular}{|l|c|c|c|c|}
\hline \multicolumn{1}{|c|}{ Pathogenes } & \multicolumn{3}{c|}{ Episodes with Certain Pathogen in Number (\%) } \\
\hline \hline S. aureus & $\mathbf{2 0 0 2}$ & $\mathbf{2 0 0 3}$ & $\mathbf{2 0 0 4}$ & $2(4.4)$ \\
\hline CoNS & $5(12.1)$ & $6(13.0)$ & $21(46.7)$ & $5(5.4)$ \\
\hline Enterobactericiae & $8(19.5)$ & $12(25.5)$ & $12(24.4)$ & $3(4)$ \\
\hline Enterococcus spp. & $17(41.4)$ & $19(40)$ & $1(2.2)$ & $12(13.0)$ \\
\hline Candida albicans & $2(4.9)$ & $5(10.6)$ & $2(4.4)$ & $5(5.4)$ \\
\hline Candida non albicans & $1(2.4)$ & $2(4.2)$ & $2(4.2)$ & $4(8.7)$ \\
\hline Total CRBSI episodes (number) & $5(12.1)$ & 47 & 92 \\
\hline
\end{tabular}

the period 2001-2005 had CRBSI. Though the rate of CRBSI is relatively high, the mortality related to CRBSI was zero during the period year 2002 to 2005 .

This study shows that the first infections occurred after 30 days of catheter installation in $92.9 \%$ of cases. Besides, we could not identify any of the risk factors registered for relapse/recurrent infections, which indicated that the prolonged use of catheter is probably the only predisposing factor for CRBSI in this population. This can only be clarified by a prospective or a case-control study.

The most predominant microorganisms found in this population were CoNS, comprising $44.7 \%$ of the CRBSI episodes. This is in agreement with the earlier published studies $[5,6,8] .31 .6 \%$ of CoNS were resistant to both methicillin and gentamicin, leading to $13.8 \%$ of all CRBSI episodes. There was an increase of CoNS CRBSI from 2002 $(19.6 \%)$ to 2005 (44.6\%), while Enterobacteriaciea and S. aureus became less dominant (Table 2). Apparently, the empirical antibiotic therapy played important role on the population of microorganisms.

The number of blood cultures increased from 2002 to 2004 annually at about $13 \%$, while from 2004 to 2005 increased at $50 \%$. This probably had contributed partly to the significant increase in the number of CRBSI episodes in 2005 (Table 2). The number of new patients per year with CRBSI was relatively constant, approximately 25 patients/year.

Candida spp. had been involved in $14.4 \%$ CRBSI episodes. Among these episodes, $61.4 \%$ had non-albicans species involved, which demand treatment with broad-spectrum anti-fungal drugs. The high prevalence of candidemia was also shown from earlier studies $[5,8]$, which is a reflection of the long-term use of intravascular catheter. It has been reported that the incidence of candidemia has increased in various parts of the world over the last decades and some species are not fully susceptible to fluconazole $[9,10]$. This implies that empiric treatment for Candida in CRBSI should be with echinocandins, liposomal amphotericin or new generation azoles.

The empirical antibiotic therapy was cefuroxime, gentamicin and metronidazol in the period 2001-2005. The cur- rent antibiotic therapy regiment for this patient group is changed to cefuroxime and ciprofloxacin. The removal of metronidazol from the empirical antibiotic therapy was guided by the fact that only $1.3 \%$ of all CRBSI episodes were caused by anaerobic bacteria. Both antibiotic regiments are able to treat most of the serious bacterial infections, such as S. aureus and Enterobacteriaceae. However, these regiments are not able to cover $95 \%$ of the predominant etiology, leaving especially a great part of the CoNS $(13.8 \%$ of all infection episodes), Enterococcus spp. (10.4\%), and Candida spp. (14.4\%) untreated. Consequently, we suggest that vancomycin and an effective anti-fungal drug, such as echinocandin, could be considered into the empirical therapy.

According to the results from this study, we strongly believe that the empiric antibiotic treatment regiment should be based on the local epidemiology data. Regular monitoring of the predominant pathogenic organisms for CRBSI in patients receiving HPN is important for adjusting the empiric treatment strategy.

\section{CONFLICT OF INTEREST}

All authors report no conflicts of interest relevant to this article.

\section{ACKNOWLEDGEMENTS}

None declared.

\section{REFERENCES}

[1] Dudrick SJ, Wilmore DW, Vars HM, Rhoads JE. Long-term total parenteral nutrition with growth, development, and positive nitrogen balance. Nutr Hosp 2001; 16: 287-92.

[2] Van GA, Messing B. Home parenteral nutrition in adults: new trends raise new questions. Nutrition 1997; 13: 479-80.

[3] Bonifacio R, Alfonsi L, Santarpia L, et al. Clinical outcome of long-term home parenteral nutrition in non-oncological patients: a report from two specialised centres. Intern Emerg Med 2007; 2: 188-95.

[4] Cicalini S, Palmieri F, Noto P, Boumis E, Petrosillo N. Diagnosis of intra vascular catheter-related infection. J Vasc Access 2002; 3: 114-9.

[5] Jeppesen PB, Staun M, Mortensen PB. Adult patients receiving home parenteral nutrition in Denmark from 1991 to 1996: who will benefit from intestinal transplantation? Scand J Gastroenterol 1998; 33: 839-46. 
[6] Ugur A, Marashdeh BH, Gottschalck I, et al. Home parenteral nutrition in Denmark in the period from 1996 to 2001. Scand J Gastroenterol 2006; 41: 401-7.

[7] Van GA, Vahedi K, Abdel-Malik, et al. Clinical, social and rehabilitation status of long-term home parenteral nutrition patients: results of a European multicentre survey. Clin Nutr 2001; 20: 205-10.

[8] Rannem T, Ladefoged K, Tvede M, Lorentzen JE, Jarnum S. Catheter-related septicaemia in patients receiving home parenteral nutrition. Scand J Gastroenterol 1986; 21: 455-60.
[9] Falagas ME, Roussos N, Vardakas KZ. Relative frequency of albicans and the various non-albicans Candida spp among candidemia isolates from inpatients in various parts of the world: a systematic review. Int J Infect Dis 2001; 14: e954-e966.

[10] Arendrup MC, Brun B, Christensen JJ, et al. National surveillance of fungemia in Denmark (2004 to 2009). J Clin Microbiol 2011; 49: $325-34$.

Received: August 03, 2012

Revised: September 18, 2012

Accepted: October 05, 2012

(C) Nielsen et al.; Licensee Bentham Open.

This is an open access article licensed under the terms of the Creative Commons Attribution Non-Commercial License (http://creativecommons.org/licenses/ by-nc/3.0/) which permits unrestricted, non-commercial use, distribution and reproduction in any medium, provided the work is properly cited. 